Urologe 2011 · 50:415

DOI 10.1007/s00120-011-2565-z

Online publiziert: 8. April 2011

(c) Springer-Verlag 2011

\author{
H. Rübben \\ Klinik und Poliklinik für Urologie, Kinderurologie \\ und Urologische Onkologie, Universitätsklinikum Essen
}

\title{
Uroonkologie 2011
}

Die Uroonkologie umfasst neben der operativen Behandlung urologischer Tumorerkrankungen auch ihre Primarprävention, Früherkennung, Diagnostik und konservative Systemtherapie ebenso wie die Nachsorge, Supportion und Palliativmedizin. Auf der letzten Jahrestagung der Deutschen Gesellschaft für Urologie war die Uroonkologie nicht nur ein wissenschaftlicher Schwerpunkt, sondern Wert gelegt wurde auch auf strukturelle Entwicklungen und deren qualitätsgesicherten Umsetzung. Dies betrifft zum einen die Durchführung dieser Maßnahmen durch die urologischen Ärztinnen und Ärzte in den Krankenhäusern sowie durch die niedergelassenen Kolleginnen und Kollegen.

Die Herausgeber von Der Urologe haben deshalb beschlossen, im Jahre 2011 in Der Urologe die Uroonkologie kontinuierlich zu beleuchten und die Ausgabe, die zur Jahrestagung erscheinen wird, der Uroonkologie zu widmen. Neben der Darstellung wichtiger neuer wissenschaftlicher Erkenntnisse werden sich die Beiträge v. a. mit deren klinischen Umset- zung befassen. Dies beinhaltet z. B. den Umgang mit neuen für die Behandlung urologischer Tumoren eingeführten Substanzen hinsichtlich Indikation, Applikation und Vermeidung sowie Behandlung von Nebenwirkungen und Komplikationen.

Wir freuen uns über Ihr Interesse an der Uroonkologie und deren kontinuierlichen Weiterentwicklung in unserem Fach zum Wohl unserer Patienten.

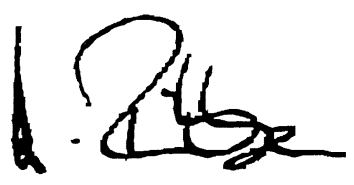

Im Namen der Herausgeber H. Rübben

\section{Korrespondenzadresse}

Prof. Dr. Dr. h. c. H. Rübben

Klinik und Poliklinik für Urologie, Kinderurologie und Urologische Onkologie, Universitätsklinikum Essen, Hufelandstraße 55, 45122 Essen herbert.ruebben@uni-duisburg-essen.de 